\title{
FGFs/FGFRs-dependent signalling in regulation of steroid hormone receptors - implications for therapy of luminal breast cancer
}

Dominika Piasecka ${ }^{1 \dagger}$, Marcin Braun $^{1 \dagger}$, Kamila Kitowska $^{2}$, Kamil Mieczkowski $^{2}$, Radzislaw Kordek $^{1}$, Rafal Sadej ${ }^{2^{*}}$ (1) and Hanna Romanska ${ }^{1 *}$

\begin{abstract}
Stromal stimuli mediated by growth factor receptors, leading to ligand-independent activation of steroid hormone receptors, have long been implicated in development of breast cancer resistance to endocrine therapy. Mutations in fibroblast growth factor receptor (FGFR) genes have been associated with a higher incidence and progression of breast cancer. Increasing evidence suggests that FGFR-mediated interaction between luminal invasive ductal breast carcinoma (IDC) and its microenvironment contributes to the progression to hormone-independence. Therapeutic strategies based on FGFR inhibitors hold promise for overcoming resistance to the ER-targeting treatment. A series of excellent reviews discuss a potential role of FGFR in development of IDC. Here, we provide a concise updated summary of existing literature on FGFR-mediated signalling with an emphasis on an interaction between FGFR and estrogen/progesterone receptors (ER/PR) in IDC. Focusing on the regulatory role of tumour microenvironment in the activity of steroid hormone receptors, we compile the available functional data on FGFRs-mediated signalling, as a fundamental mechanism of luminal IDC progression and failure of anti-ER treatment. We also highlight the translational value of the presented findings and summarize ongoing oncologic clinical trials investigating FGFRs inhibition in interventional studies in breast cancer.
\end{abstract}

Keywords: Breast cancer, Estrogen receptor, Progesterone receptor, Tumour microenvironment, Fibroblast growth factor receptors

\section{Background}

Invasive ductal breast carcinoma (IDC) is divided into biologically distinct and clinically relevant subgroups on the basis of immunohistochemical status of the estrogen receptor (ER), progesterone receptor (PR), human epidermal growth factor receptor 2 (HER2) and Ki-67 proliferation index $[1,2]$. These histopathological subclasses can also be displayed at the molecular level as intrinsic molecular

\footnotetext{
*Correspondence: hanna.romanska@gmail.com; rafal.sadej@gumed.edu.pl ${ }^{\dagger}$ Dominika Piasecka and Marcin Braun are the first authors and did equal contribution.

${ }^{2}$ Department of Molecular Enzymology, Intercollegiate Faculty of Biotechnology, University of Gdansk and Medical University of Gdansk, Debinki 1 Street, 80-211 Gdansk, Poland

1Department of Pathology, Chair of Oncology, Medical University of Lodz, Pomorska 251, 92-213 Lodz, Poland
}

subtypes, i.e. luminal A, luminal B, HER2-enriched or triple-negative IDC $[3,4]$.

The luminal A subtype represents the majority of IDC cases $(50-60 \%)$ and is defined as ER+/PR+/HER2 $-/$ Ki67 ${ }^{\text {low }}$ or highly expressing ER-related genes specified in molecular profiles (e.g. PAM50) at the protein or mRNA level, respectively $[2,3,5,6]$. The luminal B IDC, which represents $10-20 \%$ of all cases, is identified with an $\mathrm{ER}+/ \mathrm{PR}^{\text {low }} / \mathrm{HER} 2+/-/ \mathrm{Ki}^{\mathrm{h}} 7^{\text {high }}$ phenotype or a type expressing ER-related genes at low-to-moderate levels [3, 5-8]. Among all IDC subtypes, luminal A IDC patients have the best survival rate. This is due to both slow growth of the tumours and availability of ER-targeting agents such as tamoxifen, fulvestrant or aromatase inhibitors $[3,6,9]$. However, despite relatively high efficiency of anti-ER first-line endocrine therapy 
[9], approximately $45 \%$ of women do not respond to tamoxifen (de novo resistance), whereas acquired resistance to the drug develops ultimately in all tamoxifen-receiving patients, posing a serious clinical problem [10]. De novo resistance to hormone therapy is particularly frequent in patients with luminal B IDC $[8,11-13]$. Cancer cells of luminal B type are characterised by low or negative expression of PR, which as an ER-regulated gene, is thought to reflect steroid hormone-dependency and thus regarded as a predictor of responsiveness to endocrine therapy. In addition, luminal B cells express a number of ER-independent cell cycle proteins, tyrosine kinase receptors and components of their downstream signalling pathways, which render luminal B IDC partially independent of hormonal stimulation $[8,11]$. Poor responsiveness of luminal $B$ tumours to endocrine therapy was confirmed by several studies [12-16]. Investigation into the mechanisms underlying regulation of steroid hormone receptors' function and development of steroid hormone independence is, therefore, a pursuit of modern oncology.

It has been now well acknowledged that a cross-talk between tumour and its microenvironment (TME - tumour microenvironment) can promote cancer progression and development of resistance to therapy [17-22]. Stromal cells i.e. fibroblasts, immune and inflammatory cells, adipocytes and neuroendocrine cells secrete a wide range of a substances such as growth factors (e.g. FGFs, VEGF, EGF, TGF $\beta$ ), cytokines and chemokines (e.g. IL-1, IL-6, TNF $\alpha$ ) [23]. A number of TME-derived factors have been implicated in mediation of tumour - TME interaction. For example, infiltrating inflammatory cells modulated cell invasiveness by providing a 'chemotactic escape route' facilitating migration of cancer cells from the bulk of the tumour [24-26]. In breast cancer, a reciprocal paracrine loop between macrophages and cancer cells, involving EGF, CSF-1, CSF-2 or CCL18, led to the epithelial to mesenchymal transition (EMT), increased cell motility, invasion and metastasis [27, 28]. Cancer-associated fibroblasts (CAFs) are one of the most abundant cellular components of the stroma in various epithelial tumours, including breast carcinoma. Moreover, of all growth factors/cytokines secreted by CAFs, fibroblast growth factors (FGFs) emerged as the most powerful mediators of breast cancer progression, function of steroid hormone receptors and resistance to endocrine therapies [29-35].

\section{Main text}

\section{Fibroblast growth factor receptors in breast cancer}

FGFR family consists of four transmembrane receptors (FGFR1-4) containing intracellular domain with kinase activity [36-38]. There are eighteen known FGFs that bind with a different affinity to one or few members of the FGFR family [38, 39]. The signal from FGF receptors is transduced via Ras-dependent mitogen-activated protein kinase (MAPK), phosphoinositide 3-kinase (PI3K)/AKT or STATs-dependent pathways [40, 41]. In the organogenesis of the mammary gland, FGF/FGFR signalling (especially the FGF10/FGFR2 axis) controls the very early stages of steroid hormone-dependent development of the ducts as well as survival and proliferation of postnatal mammary luminal and basal epithelial cells [4244]. FGF/FGFR signalling plays a fundamental role in numerous physiological processes and its dysregulation has been associated with several developmental abnormalities and malignancies, including IDC.

Amplification/overexpression of FGFR1, FGFR2 and FGFR4 was reported as the most frequent genetic aberrancy within the FGFR family in human cancer [38, 45-47]. FGFR 1 is amplified in $8.7 \%$ of all breast cancers and this was shown as an independent predictor of overall survival [48]. Amplifications of FGFR2 and FGFR4 are rarer, observed in less than 1 and $2.3 \%$ of breast cancer patients, respectively [49]. There is a strong evidence for the association between point mutations in FGFR genes and breast cancer aggressiveness, metastasis as well as resistance to chemo- and endocrine therapy [50-55]. Moreover, several polymorphisms in FGFR2, FGFR3 and FGFR4, but not FGFR1 gene, were associated with a high risk of IDC [38, 56-65]. Biological consequences of FGFR2 polymorphism were confirmed in several meta-analyses. The ten most frequent FGFR2 polymorphisms (rs1078806, rs11200014, rs1219648, rs2420946, rs2981578, rs2981579, rs2981582, rs3135718, rs10736303, and rs3750817), out of all 23 reported in the literature, were found to be significantly associated with an increased breast cancer risk in a total of 121,740 cases and 198,549 controls recruited for the biggest of the study $[15,57-59,61-63,65,66]$. Interestingly, polymorphisms in FGFR2 (rs2981582, rs1219648, and rs2420946) were characterised by a strong association with the risk of ER-positive but not ER-negative IDC [67, 68]. This was additionally confirmed in the meta-analysis by Wang et al., which involved 288,142 participants of 37 studies [62]. FGFR4 rs351855 was repeatedly reported to be associated not only with a higher risk of breast cancer, but also with its aggressiveness and resistance to anti-ER treatment $[38,50,51]$. Functional studies revealed that polymorphisms in FGFRs most commonly consist of missense mutations, which result in either alteration of the structure of ligand-binding domain or constitutive activation of FGFR kinase domain $[38,50,51,53]$. The specificity to luminal IDC relates also to the genetic polymorphism of the FGFR ligands. FGF10 (one of FGFR2 ligands) rs10941679 was associated with a higher risk of luminal IDC and reported to result in overexpression of FGF10 and hyperactivation of the FGFR2 pathway in ER-positive IDC cells [65].

Over the last few years FGFR genes have emerged as important players in the pathogenesis of diverse carcinomas, including luminal IDC. This review summarizes 
for the first time the existing experimental and clinical data on the cross-talk between steroid hormone receptors and the FGFs/FGFRs axis in view of their relevance to ER-targeting therapy.

\section{ER and PR - signalling pathways and regulation}

Estrogen and progesterone are essential regulators of mammary gland development. Estrogen is strongly involved in a process of ducts' formation, whereas progesterone promotes growth of the gland lobules [69]. Progesterone and PR work in concert with estrogen and ER to induce expansion of glandular structures during organogenesis of the breast [70]. In addition to their physiological role, both receptors and their cognate ligands have been implicated in development and progression of luminal IDC. ER and PR belong to the nuclear receptors' family of ligand-activated transcription factors, which regulate genes expression by activation or repression of transcription [71, 72]. Binding of steroid hormones induces receptor dimerization and subsequent conformational changes, which in turn expose nuclear localization signal within the receptor. This is followed by receptor translocation to the nucleus, where it binds to DNA sequences and enhances or silences transcription of target genes. This "classical" pathway of the steroid hormone receptor-mediated signalling is characterised by ER/PR binding to specific genomic sequences i.e. ERE - estrogen and PRE - progesterone responsive element, respectively, and results in the interaction of the receptors with co-regulators to modulate target genes expression (Figs. 1a and 2a). In addition to the conventional steroid hormone-dependent control of ER/PR activity, their reciprocal regulation and cross-talk with various signalling pathways, triggered by growth factor receptors, affect their function. It has been recently shown that, upon progesterone stimulation, PR interacted with ER and recruited it away from the classical ER-binding sites to the new PR-directed locations, resulting in an activation of a set of genes associated with a good clinical outcome (Fig. 3) [73, 74]. As demonstrated for the first time by Denner and co-workers, PR can be phosphorylated and transcriptionally activated independently of progesterone binding [75]. Several reports confirmed growth factor receptors-mediated activation of steroid hormones receptors in the absence of their cognate ligands. It was shown that heregulin treatment of luminal IDC cells resulted in transactivation of PR and this required both functional ErbB2 and MAPK activity [76]. PR was proved to be activated also by other growth factors such as IGF-1 (insulin-like growth factor-1) [77], EGF (epidermal growth factor) [78], FGF2 [79] and FGF7 [80]. Similarly, IGF-1 [81, 82], EGF [83-85] and FGF7 [32] were reported to activate ER in a ligand-independent manner. There is an evidence to suggest that growth factor-mediated ER or PR activation results in steroid hormone receptor phosphorylation followed by its ubiquitination and degradation [86-89]. Moreover, growth factor-dependent activation of MAPK and PI3K (phosphatidylinositol-3-kinase)/AKT leads to ER phosphorylation resulting in cell resistance to tamoxifen [84, 90]. Phosphorylation of PR in response to EGF-triggered signalling can negatively regulate progesterone-induced PR sumoylation $[78,91]$. This posttranslational modification by small ubiquitin-like modifier SUMO was previously shown to stabilize PR and inhibit PR transcriptional activity [92]. Consistently, recent studies performed in luminal IDC cells clearly demonstrated that FGF7/FGFR2-triggered phosphorylation of PR at Ser294, followed by PR ubiquitination and receptor degradation via the $26 \mathrm{~S}$ proteasome pathway [80], contributed to the progression towards a steroid hormone-independent phenotype. Phosphorylated and desumoylated PR is thought to be transcriptionally hyperactive, rapidly turned over and thus difficult to detect (e.g. by routine immunohistochemistry) [78, 91]. If so, it is likely that such a hyperactive and rapidly degraded PR might actually be present in breast tumours clinically classified as PR-low or PR-negative (luminal B IDC). Knutson and co-workers have recently confirmed that phospho-PR Ser294 and elevated expression of a unique phospho-PR genes signature was detected in a substantial subset of phenotypically PR-negative tumours [93].

Taken together, these results demonstrate that signalling triggered by stroma-derived growth factors, which targets ER/PR, might represent a mechanism of IDC progression towards more aggressive steroid hormone-independent phenotype, contributing to the failure of the anti-ER therapies.

\section{Fibroblast growth factor receptors-dependent signalling and regulation of steroid hormone receptors}

A number of studies have unequivocally demonstrated a functional link between FGFRs and steroid hormone receptors. Back in 1998, McLeskey and colleagues, using MCF7-derived cell lines overexpressing FGF1 or FGF4, showed that FGFs were able to replace estrogen as a mitogenic stimulus indispensable for ER-positive tumour growth. Thus, FGFs-dependent signalling bypasses the ER signal transduction pathways and might be responsible for poor response to anti-ER treatments with tamoxifen or fulvestrant [94]. On the other hand, overexpression of FGF8b (a preferential ligand of FGFR1IIIc and FGFR2IIIc splice isoforms as well as FGFR4) in MCF7 luminal IDC cell line led to an increase of anchorage-independent growth and provided an additional growth advantage for cells stimulated with estradiol. FGF8b-overexpression also promoted MMP9 secretion and IDC cell invasion. FGF8b-transfected cells xenografted into nude mice formed faster growing and more densely vascularized 


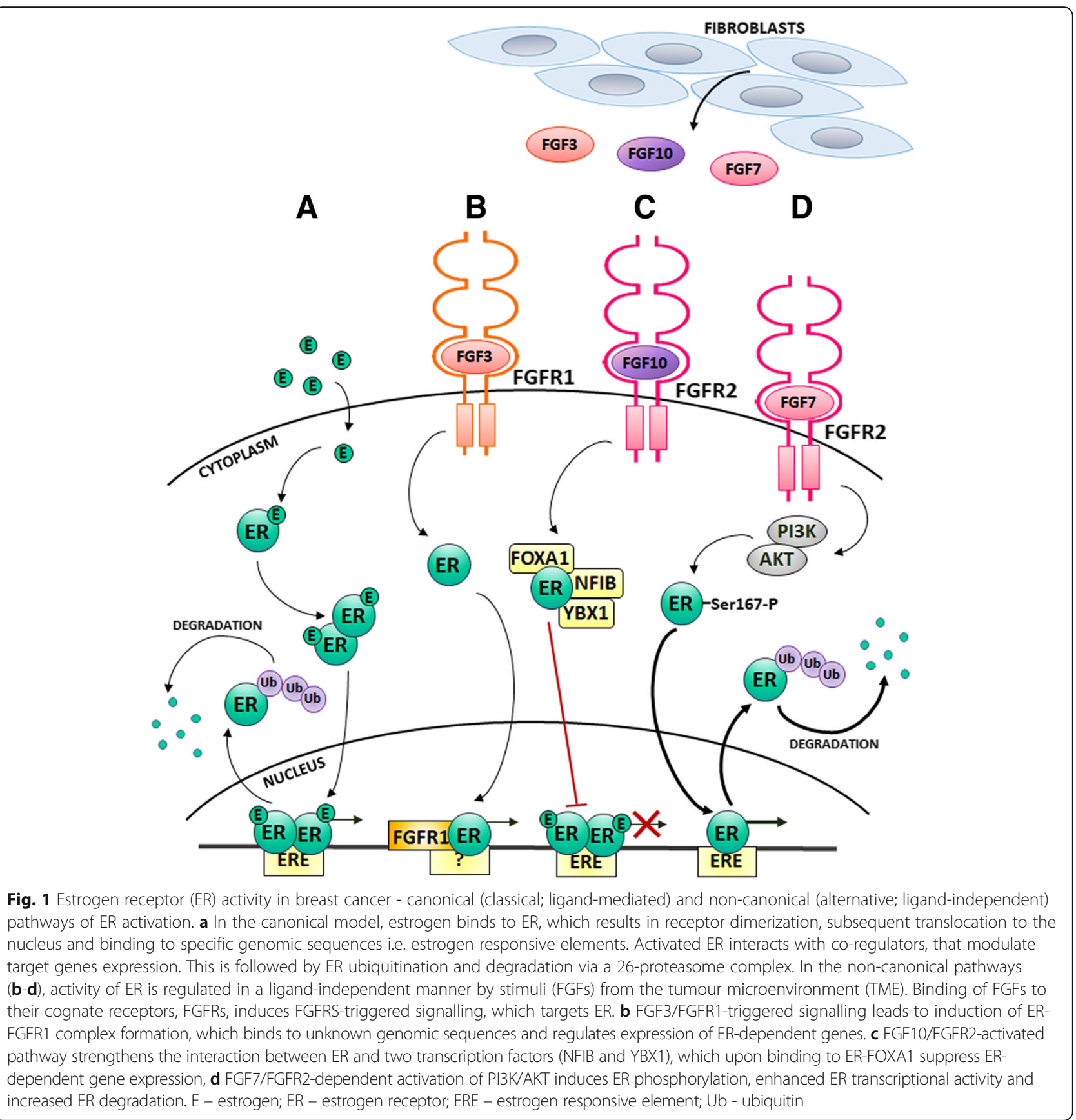

tumours [95]. FGFR1 amplification and overexpression was frequently found in ER-positive/PR-negative IDC tissue, indicating that FGFR1 is strongly associated with worse prognosticating luminal B IDC. Indeed, FGF2/FGFR1-triggered signalling in luminal BCa cell lines with FGFR1 amplification and overexpression was shown to inhibit ER-directed transcription, which was reflected by suppression of PR expression [96]. It was demonstrated that FGFR1 is amplified/overexpressed in $43 \%$ of ER-positive IDC patients resistant to aromatase inhibitor (letrozole). Interestingly, overexpression of FGFR1 was accompanied by upregulation of FGF3, FGF4 and FGF19. Long-term estrogen-deprivation of CAMA1 luminal BCa cell line, mimicking therapy with letrozole, resulted in increased FGFR1-ER interaction, which required FGFR1 kinase activity. This led to the estrogen-independent induction of ER-regulated genes, which was confirmed by ChIP-seq analysis. Further studies revealed that FGF3 treatment shifted ER and FGFR1 binding to the new chromatin regions, unoccupied in the absence of the FGFRs' ligands (Fig. 1b). Combined inhibition of ER and FGFR with fulvestrant and lucitanib, respectively, abrogated ER or FGFR1 binding to 


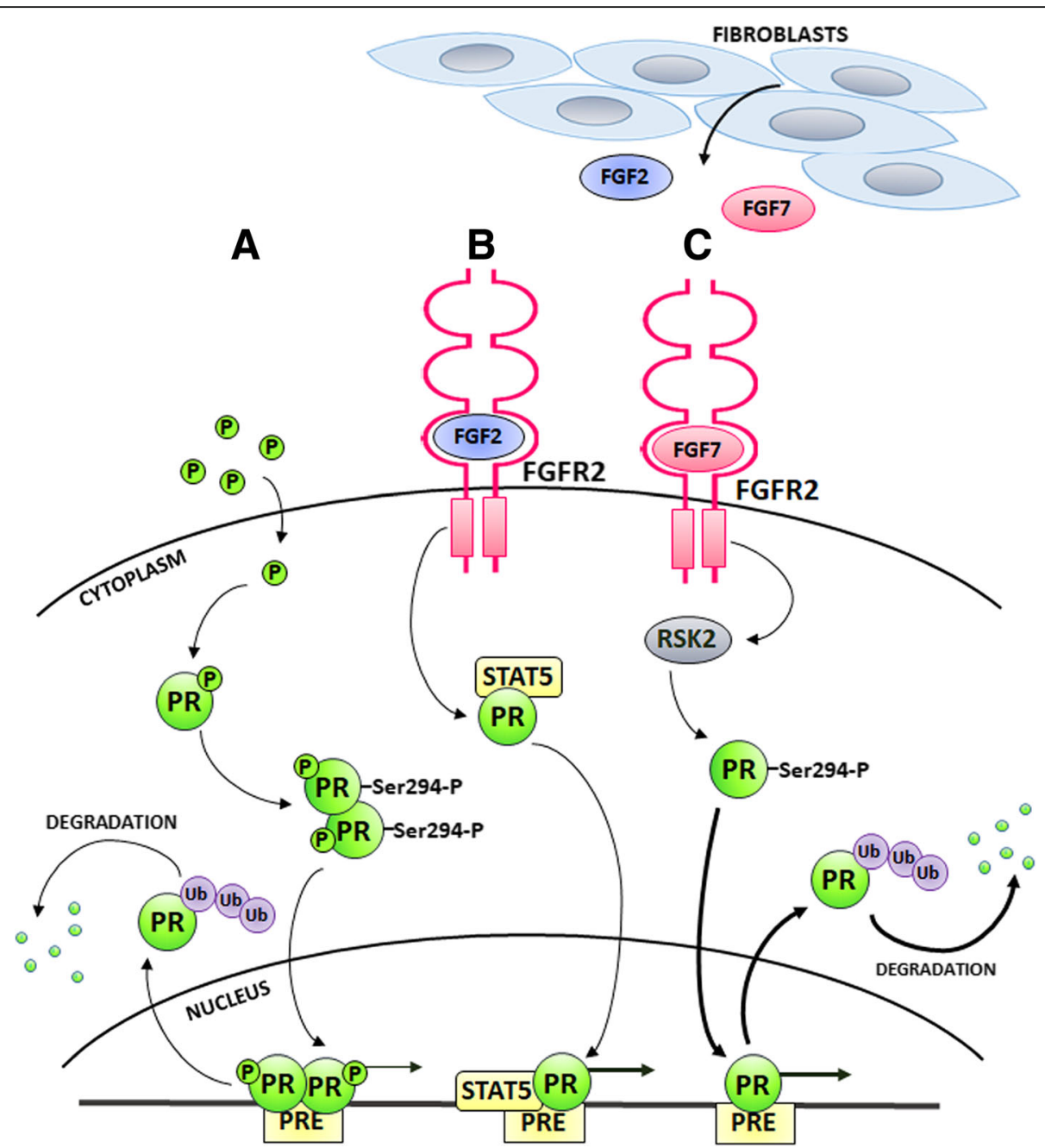

Fig. 2 Progesterone receptor activity in breast cancer - canonical (classical; ligand-mediated) and non-canonical (alternative; ligand-independent) pathways of PR activation. a In the classical model, progesterone binds to PR, which induces receptor dimerization, translocation to the nucleus and binding to PR specific genomic sequences i.e. progesterone responsive elements. This results in regulation of expression of PR-dependent genes, followed by PR ubiquitination and proteasomal degradation. In the non-canonical pathways (b-c), PR activation is induced by tyrosine kinases. FGFRs mediate a tumour microenvironment-originated signal (FGFs), which targets PR. b FGF2/FGFR2 signalling leads to PR colocalization with STAT5 in a nucleus of cancer cells, which stimulates transcription of PRE-containing genes. c FGF7/FGFR2-triggered signalling increases transcriptional activity of PR via RSK2-mediated PR phosphorylation at Ser294 and subsequent PR ubiquitination and degradation in proteasome. P - progesterone; PR - progesterone receptor; PRE - progesterone responsive element, Ub - ubiquitin

these sites, suggesting that FGF/FGFR pathway modulates ER-DNA interaction. In addition, combination of these inhibitors strongly impaired growth of ER-positive IDC with FGFR1 amplification. Profiling by qRT-PCR of ER-positive/ FGFR1-amplified IDCs, deprived of estrogen and treated with FGF3/FGF19, identified a subset of ER-responsive genes, which included TFF1, CCND1, THSB1, CTGF, CCL2 and EGR3. In addition, gene-set enrichment analysis (GSEA) from letrozole-treated IDC patients revealed, that ER-related pathways were still active in ER-positive/ FGFR1-amplified primary tumours. This suggests that association of FGFR1 with ER maintains ligand-independent ER transcription and mediates resistance to estrogen deprivation in ER-positive IDC [97]. FGFR1 amplification has been shown as an independent negative prognostic factor for disease-free and overall survival exclusively in patients with ER-positive IDC. Chromogenic in situ hybridisation indicated that breast cancer patients with FGFR1 amplification in the ER-positive group were characterised by lack of PR expression and were at a significantly higher risk for development of distant metastases [48].

FGFR2 activation by FGF10, the most potent FGFR2 ligand in mammary epithelial cells [98], was reported to counteract estrogen-triggered ER-dependent signalling [99]. Further analysis showed that three breast cancer risk SNPs (rs2981578, rs35054928 and rs45631563) in the 


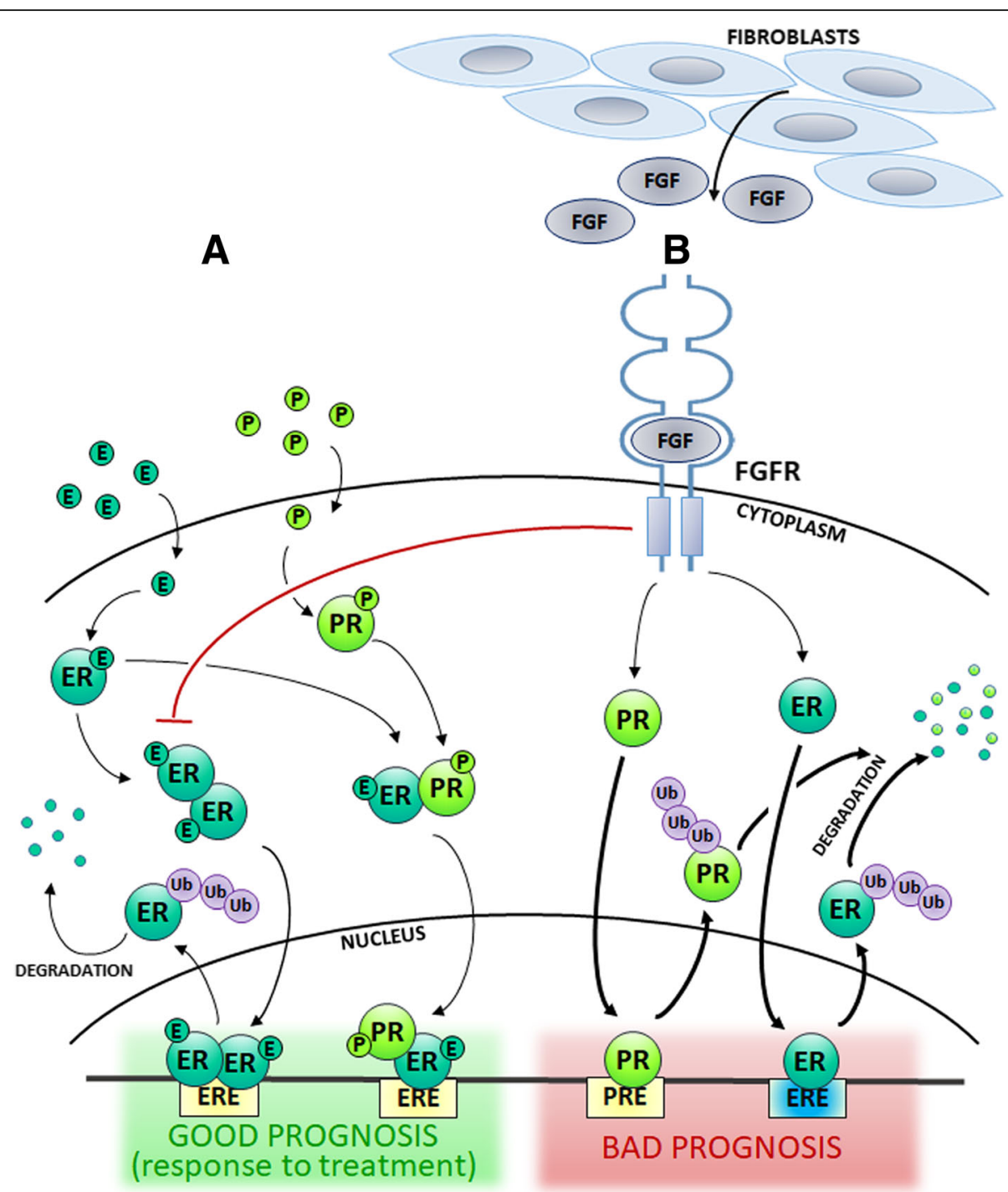

Fig. 3 Ligand-dependent and -independent activation of ER/PR - an impact on patient prognosis in luminal IDC. a ER is activated in response to estrogen. In addition, progesterone induces PR/ER dimerization and recruits ER away from the classical ER-binding sites to the new PR-directed sites, promoting expression of a gene set associated with GOOD PROGNOSIS. $\mathbf{b}$ There are two major mechanisms of FGFRs-induced steroid hormone-independent ER/PR regulation, both associated with POOR PROGNOSIS: a FGFRs-triggered shift in ER binding to DNA (ERE, in blue), and FGFRs-dependent rapid activation of ER and PR leading to their subsequent degradation. E - estrogen; ER - estrogen receptor; ERE - estrogen responsive element; $\mathrm{P}$ - progesterone; $\mathrm{Ub}$ - ubiquitin

FGFR2 locus were responsible for reduced expression of FGFR2, conferred increased estrogen responsiveness and a higher risk of ER-positive IDC [99]. This would suggest that reduced expression of FGFR2 (due to specific polymorphism in FGFR2 gene) associates with good prognosis. There are data clearly indicating FGFR2 involvement in progression towards ER-negative luminal IDC, a subtype more aggressive and less responsive to the treatment. FGF10/FGFR2 signalling was shown in MCF7 and ZR-75-1 IDC cell lines to strengthen the interaction of ER with two transcription factors, NFIB and YBX1. Binding to the ER-FOXA1 complex, both factors repressed ER target gene expression (Fig. 1c). This suggests that FGFR2 might have a broad effect promoting IDC progression towards estrogen-independent basal-like phenotype and application of FGFR inhibitors could increase tumour sensitivity to anti-ER therapies [100]. Moreover, as demonstrated by our group, treatment of MCF7 and T47D cells with FGF7 or CAFs-conditioned media induced ER ubiquitination and subsequent ER degradation in proteasome. This was mediated by FGFR2-induced PI3K/AKT signalling pathway, which enhanced ER-Ser 167 phosphorylation (Fig. 1d). FGFR2-induced ER loss in response to FGF7 and/or CAFs-derived signals in the cell lines was 
corroborated by an inverse correlation between FGFR2 and ER expression in tissue from IDC patients [32]. A study carried out in a murine model of MPA (medroxyprogesterone acetate - synthetic progestin)-induced mammary carcinoma has revealed that hormone-independent (HI) tumours were characterised by a higher level of FGFR2 expression than their hormone-dependent counterparts. In addition, CAFs isolated from $\mathrm{HI}$ tumours were shown to secrete FGF2, which led to phosphorylation of PR (at Ser190 and Ser294) and hormone-independent growth in both HI and luminal IDC (T47D) cells. This effect was abolished by application of PD173074, a FGFRs' inhibitor, or FGF2 neutralising antibodies [31]. The same group has further demonstrated that FGFR2 co-localized with STAT5 and PR in a nucleus of luminal IDC cells in response to treatment with FGF2 and MPA (Fig. 2b). This nuclear interaction, associated with increased transcription of PRE-containing reporter genes, was also observed in human IDC tissue [79]. In addition, exogenously administered FGF2 was able to mimic MPA, and this effect was reverted by the antiprogestin, RU486 [31, 101]. In our recent study, we found that regulation of activation and turnover of PR was FGFR2-dependent. FGF7/FGFR2-triggered signalling led to PR phosphorylation at Ser294 and subsequent PR ubiquitination and degradation in proteasome. RSK2 kinase was identified as a mediator of FGFR2 action towards PR loss (Fig. 2c). Immunohistochemical analysis of IDC tissue specimens demonstrated that expression of PR inversely correlated with that of an active form of RSK (RSK-P). Patients with RSK-P(+)/PR(-) tumours had a higher risk of recurrence, when compared with the rest of the cohort. These results indicate that the FGFR2-RSK2 signalling pathway activates PR and regulates its turnover, which might contribute to the TME-driven progression of luminal IDC towards steroid hormone-independence [80].

FGFRs are not only the powerful regulators of steroid hormone receptors function but, as shown for FGFR3, they may also act as effective mediators of ER activity. FGFR3 was identified as a key facilitator of ER-driven expansion of breast cancer stem cells (BCSCs). Analyses of antibody-based protein arrays revealed that estrogen treatment induced secretion of FGF family members i.e. FGF2, FGF4, FGF6, FGF7 and FGF9 in MCF7 cell line. Further in vitro and in vivo studies showed that estrogen stimulation led to the expansion of functional BCSCs pool through a paracrine FGF9/FGFR3/Tbx3 signalling. This suggests that FGF9/FGFR3/Tbx3-mediated promotion of BCSCs' survival and growth might be one of the mechanisms responsible for the failure of treatment, including ER-targeting therapies [52].

These studies demonstrate that in response to stromal stimuli, FGF/FGFR signalling not only regulates steroid hormone receptors turnover, but also determines their transcriptional activity and DNA-binding, which might contribute to IDC progression towards steroid hormoneindependence.

\section{CAFs/FGFs/FGFRs and response to anti-ER treatment}

There is growing evidence that regulation of ER and PR function by tumour microenvironment contributes to breast cancer progression. Stromal cells were shown to upregulate aromatase expression and increase estrogen levels in the tumour [102]. An impact of CAFs on response to endocrine therapy has been demonstrated in numerous studies. For example, co-culture of premalignant mammary cells (EIII8, a subclone of MCF10A) or invasive IDC cells (MCF7) with fibroblasts derived from ER/PR-positive tumours enhanced inhibitory effect of tamoxifen on cell growth in 3D cultures, whereas fibroblasts from of ER/PR-negative tumours triggered an opposite effect i.e. promoted acquisition of resistance to tamoxifen [35]. Recent study suggested that effectiveness of tamoxifen and patients' outcome in luminal IDC are determined by CAFs' phenotype. In the presence of CD146-negative CAFs, MCF7 cells implanted into mice displayed decreased ER expression, diminished sensitivity to estrogen and increased resistance to tamoxifen. Conversely, CD146-positive CAFs led to a sustained ER expression, estrogen-dependent proliferation and sensitivity to tamoxifen [29]. In addition, in ER-positive IDCs, existence of CAFs subpopulation with low level of ERK phosphorylation was associated with worse response of patients to tamoxifen-based therapy. This suggests that a status of ERK phosphorylation in CAFs might be used as a biomarker of efficiency of anti-ER treatment [103].

Being a rich source of FGFs in tumour stroma [31, 79, 104], CAFs influence luminal IDC response to endocrine therapy through FGFs/FGFRs-mediated regulation of steroid hormone receptors. Results of several studies in a murine model of estradiol-dependent breast carcinoma and human ER-positive IDC cell lines demonstrate that CAFs protect cancer cells from tamoxifen-induced cell death via activation of AKT and MAPK pathways, which leads to ER phosphorylation [32, 33, 35]. Both FGF7 and CAF-conditioned medium counteracted tamoxifen-dependent growth inhibition and this involved FGFR2 activity [32]. An association between FGFs/FGFRs axis and resistance to tamoxifen was first demonstrated twenty years ago in MCF7 cells overexpressing FGF1 and FGF4, xenografted into nude mice $[105,106]$. This was further confirmed by Turner et al. showing that FGFR1-amplified cell lines (MDA-MB-134 and SUM44) displayed resistance to tamoxifen. FGF2/ FGFR1 signalling was suggested to overcome tamoxifeninduced growth arrest and apoptosis, which has been linked with high MAPK and AKT activity as well as increased level of cyclin D1. Poor prognosis of patients 
with FGFR1-overexpressing tumours subjected to adjuvant tamoxifen-based therapy verified results of experimental studies. FGFR1 signalling was shown to suppress PR expression in vitro and this was confirmed by demonstration of an inverse correlation between FGFR1 and PR in human breast cancer tissue [96]. A novel mechanism of resistance to endocrine therapies in ER-positive IDC with FGFR1 amplification was proposed by Formisano et al. Long-term estrogen-deprivation of FGFR1-overexpressing CAMA1 luminal IDC cell line, mimicking effect of aromatase inhibitors, enhanced FGFR1 interaction with ER, leading to the induction of expression of ER-dependent genes. Simultaneous inhibition of FGFR1 and ER (with lucitanib and fulvestrant) supressed cell growth in vitro and in a PDX (patient derived xenograft) model more potently than when the drugs were administered separately. This implies that patients with endocrine resistant ER-positive/ FGFR1-amplified tumours may benefit from the treatment with combination of ER and FGFR antagonists [97]. This finding was supported by genomic profiling of 155 early ER-positive IDCs exposed to short-term estrogen suppression with letrozole, which identified amplification of FGFR1 and CCND1 (cyclin D1 gene) as a likely mechanism of resistance to the treatment. FGFR1/CCND1 co-amplification led to a greater enrichment of cell cycle genes than enhancement caused by single amplifications, which is consistent with activation of alternative mechanisms of escape from canonical cell cycle control. Furthermore, combined inhibition of FGFR1 and CDK4/6 in CAMA1 cell line abolished anti-estrogen resistance suggesting that an interaction between FGFR1 and cyclin D1 might drive estrogen-independent proliferation in co-amplified tumours [107]. FGFR2-dependent signalling was proved to counteract negative effect of tamoxifen on T47D and MCF7 cell growth with molecular mechanism involving PI3K/AKT pathway and regulation of $\mathrm{Bcl}-2$ expression [32]. Resistance to tamoxifen has also been associated with increased expression of FGFR3. The FGF1/FGFR3 axis conferred resistance to both tamoxifen and fulvestrant in an ER-independent manner (no activation of ER was observed) in MCF7 cell line. The mechanism of FGFR3-promoted proliferation of tamoxifen resistant cells relied on activation of PLC $\gamma /$ PI3K and MAPK pathways, however, inhibition of only the former resulted in reversal of the tamoxifen-resistant phenotype [108]. An elevated level of FGFR4 mRNA was reported as an independent predictor of little clinical benefit and shorter progression-free survival in IDC patients treated with tamoxifen [109].

In summary, presented studies demonstrate that microenvironmental stimuli from specific CAFs subpopulations may act as a dual-face regulator of resistance to endocrine therapy. Co-operation of hormone receptors with FGF/ FGFR-triggered signalling pathway might be an important mediator of steroid hormone independence.

\section{Therapeutic targeting and future perspectives}

The described findings demonstrate that steroid hormone-independent shift in ER binding to DNA or induction of rapid ER/PR activation triggered by FGFR are followed by ER and PR degradation (Fig. 3). This implicates that FGF/FGFR signalling pathway acts as an essential regulator of steroid hormone receptors activity. It mediates resistance to endocrine therapy induced by microenvironmental stimuli. The FGF/FGFR axis is, therefore, a promising target for therapy of luminal IDC [38, 110, 111].

The established strategies for inhibition of the FGFR/ FGF pathway fall into three main categories/classes: 1) non-selective tyrosine kinase inhibitors, which act against the intracellular domains of not only FGFRs, but also VEGFRs or PDGFRs; 2) selective inhibitors of FGFRs, which target all FGFR1-3 (due to strong similarity of structure within the receptor family, no selective inhibitors for individual FGFRs are available) or FGFR4; 3) monoclonal antibodies that either block FGFRs or entrap their ligands (reviewed in 38, 110). According to clinicaltrials.gov, there have been 179 completed or ongoing oncologic clinical trials investigating FGFs/FGFRs inhibition in interventional studies, eighteen of which concern breast cancer patients (phase I and II, NCT numbers: NCT03238196, NCT00958971, NCT020536 36, NCT01202591, NCT02202746, NCT03344536, NCT 01791985, NCT02619162, NCT01795768, NCT02511 847, NCT02915172, NCT01594177, NCT02465060, NCT02052778, NCT01928459, NCT03514121, NCT023 93248, NCT03583125).

Dovitinib (TKI258, Novartis) is an example of a non-selective inhibitor of FGFR family showing high potency for c-KIT, CSF-1, VEGFR and PDGFR which has been tested in six Phase I/II clinical trials involving advanced breast cancer patients $[38,112]$. Musolino et al. showed cautiously promising efficacy - complete and partial responses - after dovitinib administration in advanced, hormone-resistant ER-positive, HER2-negative, FGF-amplified breast cancer patients [113]. Cheng et al. reported an almost complete response (including brain lesions) to pazopanib - another multikinase inhibitor in a patient with hormone-resistant ER-positive, HER2-negative and FGFR1-amplified IDC [114]. Lenvatinib (E7080, Eisai) is another non-selective RTK inhibitor, which targets FGFR1-4, VGFR1, PDGFR, RET and KIT and was reported as a promising drug for aggressive, triple-negative breast cancer patients [115]. AZD4547, NVP-BGJ398 and JNJ-42756493 belong to the second class of selective FGFR inhibitors, and are currently under a Phase I/II clinical trial to evaluate their activity in patients with amplified FGFR1 and FGFR2 breast, squamous lung and stomach cancers $[38,116]$. In breast cancer, both NVP-BGJ398 and AZD4547 showed promising results in studies by Smyth et al. and Nogova et al., 
where patients with advanced breast cancers responded partially to their application $[117,118]$. Monoclonal antibodies are the third major class of FGF/FGFR-targeting agents. Numerous antibodies have been developed, however the knowledge about their clinical potential is limited to only a few (reviewed in 38, 111). FPA144 and MFGR1877S, monoclonal antibodies to FGFR3 and FGFR2, respectively, and FP-1039, a FGF2 trap, showed promising activities with acceptable toxicity in advanced solid tumours [119-121]. Clinical trials of anti-FGFR monoclonal antibodies specifically in breast cancer patients have not been reported, yet.

Anti-FGF/FGFR agents are tested in clinical trials either alone or, more commonly, in combination with other therapies (with standard therapies, immunotherapy or other targeted therapies) [111]. In luminal breast cancer, the most promising possibility is to combine FGFR inhibitors with anti-ER therapies. Results reported by Musolino et al. encouraged development of new trials testing such combinations (fulvestrant, palbociclib and erdafitinib in NCT03238196, AZD4547 and fulvestrant NCT01202591, Debio 1347 and fulvestrant in NCT03344536, AZD4547 and Anastrozole or Letrozole in NCT01791985, nintedanib and letrozole in NCT02619162) [113]. No multiple therapies combining FGFR inhibitors with immunotherapy (unlike in HER2-positive breast cancer - PA150-001 with pembrolizumab (NCT03514121) or afatinib together with trastuzumab and chemotherapy (NCT01594177)) have been developed yet.

In spite of an undisputed role of FGF/FGFR signalling in cancer progression, potential benefits of their clinical use are accompanied with downsides such as side effects. These include hyperphosphatemia, skin and eye dryness, keratopathy, asymptomatic retinal pigment epithelial detachment, hypertension, proteinuria, cardiac, vascular or liver impairment, diarrhoea and fatigue nausea $[38,111]$. In addition, as pertinent to all targeted therapies, various hurdles, particularly those related to tumour heterogeneity (an existence of only a subset of sensitive/responsive cells), acquired resistance, identification of predictive markers for appropriate selection of patients, need to be overcome before their routine implementation in clinic is granted. Results from early clinical trials hold promise for therapeutic efficiency of anti-FGF/FGFR agents as a complementary strategy in ER-positive breast cancer. Further functional studies are required so their use can bring lasting therapeutic benefit.

\section{Conclusion}

Prognosis of luminal IDC largely depends on cell response to endocrine therapy. This relies on expression of hormone receptors (ER/PR) and 'addiction` of cancer cells to steroid hormones. Functional studies and clinical analyses provide ample evidence that activity of ER and PR is affected by stroma-derived stimuli mediated FGFRs. FGFRs-triggered signalling can lead to emergence of steroid hormone independence and progression towards ER/ PR-negative IDC. These findings open new avenues for development of new therapeutic strategies based on FGFR inhibitors, likely to overcome resistance to commonly applied ER-targeting regimens.

\section{Abbreviations \\ BCa: Breast carcinoma; BCSC: Breast cancer stem cells; CAFs: Cancer- associated fibroblasts; ER: Estrogen receptor; ERE: Estrogen responsive element; FGF: Fibroblast growth factor; FGFR: Fibroblast growth factor receptor; HI: Hormone independence; IDC: Invasive ductal carcinoma; MAPK: Mitogen-activated protein kinase; PI3K: Phosphoinositide 3-kinase; PR: Progesterone receptor; PRE: Progesterone responsive element; TME: Tumour microenvironment}

\section{Acknowledgements}

Not applicable.

\section{Funding}

This study was supported by grants from the National Science Centre (UMO2017/27/B/NZ3/01474 to RS and UMO-2015/17/B/NZ4/02157 to RK) and Medical University of Lodz (502-03/1-034-03/502-14-329 and 502-03/1-03403/502-14-330 for DP and MB).

\section{Availability of data and materials Not applicable.}

\section{Authors' contributions}

$H R$ and RS provided direction and guidance throughout the preparation of this manuscript. DP, MB, KK and KM wrote and edited the manuscript. DP prepared all Figs. RK, RS and HR reviewed and made significant revisions of the manuscript. All authors read and approved the final manuscript.

Ethics approval and consent to participate

Not applicable.

Consent for publication

Not applicable.

\section{Competing interests}

The authors declare that they have no competing interests.

\section{Publisher's Note}

Springer Nature remains neutral with regard to jurisdictional claims in published maps and institutional affiliations.

Received: 20 March 2019 Accepted: 17 May 2019

Published online: 29 May 2019

\section{References}

1. Vollenweider-Zerargui L, Barrelet L, Wong Y, Lemarchand-Beraud T, Gomez F. The predictive value of estrogen and progesterone receptors' concentrations on the clinical behavior of breast cancer in women. Clinical correlation on 547 patients. Cancer. 1986;57(6):1171-80.

2. Sinn HP, Kreipe H. A brief overview of the $\mathrm{WHO}$ classification of breast tumors, 4th edition, focusing on issues and updates from the 3rd edition. Breast Care (Basel). 2013;8(2):149-54.

3. Perou CM, Sorlie T, Eisen MB, van de Rijn M, Jeffrey SS, Rees CA, et al. Molecular portraits of human breast tumours. Nature. 2000;406(6797): 747-52

4. Sørlie T, Perou CM, Tibshirani R, Aas T, Geisler S, Johnsen H, et al. Gene expression patterns of breast carcinomas distinguish tumor subclasses with clinical implications. Proc Natl Acad Sci U S A. 2001;98(19):10869-74. 
5. Fan C, Oh DS, Wessels L, Weigelt B, Nuyten DS, Nobel AB, et al. Concordance among gene-expression-based predictors for breast cancer. N Engl J Med. 2006;355(6):560-9.

6. Parker JS, Mullins M, Cheang MC, Leung S, Voduc D, Vickery T, et al. Supervised risk predictor of breast cancer based on intrinsic subtypes. J Clin Oncol. 2009:27(8):1160-7.

7. Dai X, Li T, Bai Z, Yang Y, Liu X, Zhan J, et al. Breast cancer intrinsic subtype classification, clinical use and future trends. Am J Cancer Res. 2015;5(10): 2929-43.

8. Nielsen TO, Parker JS, Leung S, Voduc D, Ebbert M, Vickery T, et al. A comparison of PAM50 intrinsic subtyping with immunohistochemistry and clinical prognostic factors in tamoxifen-treated estrogen receptor-positive breast cancer. Clin Cancer Res. 2010;16(21):5222-32.

9. Fisher B, Costantino J, Redmond C, Poisson R, Bowman D, Couture J, et al. A randomized clinical trial evaluating tamoxifen in the treatment of patients with node-negative breast cancer who have estrogen-receptor-positive tumors. N Engl J Med. 1989;320(8):479-84.

10. Clarke R, Liu MC, Bouker KB, Gu Z, Lee RY, Zhu Y, et al. Antiestrogen resistance in breast cancer and the role of estrogen receptor signaling. Oncogene. 2003;22(47):7316-39.

11. Creighton CJ. The molecular profile of luminal B breast cancer. Biologics. 2012;6:289-97.

12. Braun L, Mietzsch F, Seibold P, Schneeweiss A, Schirmacher P, Chang-Claude J, et al. Intrinsic breast cancer subtypes defined by estrogen receptor signalling-prognostic relevance of progesterone receptor loss. Mod Pathol. 2013;26(9):1161-71.

13. Cheang MC, Chia SK, Voduc D, Gao D, Leung S, Snider J, et al. Ki67 index, HER2 status, and prognosis of patients with luminal B breast cancer. J Natl Cancer Inst. 2009;101(10):736-50.

14. Bardou VJ, Arpino G, Elledge RM, Osborne CK, Clark GM. Progesterone receptor status significantly improves outcome prediction over estrogen receptor status alone for adjuvant endocrine therapy in two large breast cancer databases. J Clin Oncol. 2003;21(10):1973-9.

15. Arpino G, Weiss H, Lee AV, Schiff R, De Placido S, Osborne CK, et al. Estrogen receptor-positive, progesterone receptor-negative breast cancer: association with growth factor receptor expression and tamoxifen resistance. J Natl Cancer Inst. 2005:97:1254-61.

16. Prat A, Cheang MC, Martin M, Parker JS, Carrasco E, Caballero R, et al. Prognostic significance of progesterone receptor-positive tumor cells within immunohistochemically defined luminal a breast cancer. J Clin Oncol. 2013; 31(2):203-9.

17. Trédan O, Galmarini CM, Patel K, Tannock IF. Drug resistance and the solid tumor microenvironment. J Natl Cancer Inst. 2007;99(19):1441-54.

18. Son B, Lee $\mathrm{S}$, Youn H, Kim E, Kim W, Youn B. The role of tumor microenvironment in therapeutic resistance. Oncotarget. 2017;8(3):3933-45.

19. Sun Y. Tumor microenvironment and cancer therapy resistance. Cancer Lett. 2016;380(1):205-15.

20. Balkwill FR, Capasso M, Hagemann T. The tumor microenvironment at a glance. J Cell Sci. 2012;125(Pt 23:5591-6.

21. Hu M, Polyak K. Microenvironmental regulation of cancer development. Curr Opin Genet Dev. 2008;18(1):27-34.

22. Polyak K, Haviv I, Campbell IG. Co-evolution of tumor cells and their microenvironment. Trends Genet. 2009;25(1):30-8.

23. Chen F, Zhuang $X$, Lin L, Yu P, Wang Y, Shi Y, et al. New horizons in tumor microenvironment biology: challenges and opportunities. BMC Med. 2015; 13(1):45.

24. Lin EY, Pollard JW. Macrophages: modulators of breast cancer progression. Novartis Found Symp. 2004;256:158-68; discussion 68-72, 259-69.

25. Qiu SQ, Waaijer SJH, Zwager MC, de Vries EGE, van der Vegt B, Schroder CP. Tumor-associated macrophages in breast cancer: innocent bystander or important player? Cancer Treat Rev. 2018;70:178-89.

26. Aras S, Zaidi MR. TAMeless traitors: macrophages in cancer progression and metastasis. Br J Cancer. 2017;117(11):1583-91.

27. Wyckoff J, Wang W, Lin EY, Wang Y, Pixley F, Stanley ER, et al. A paracrine loop between tumor cells and macrophages is required for tumor cell migration in mammary tumors. Cancer Res. 2004;64(19):7022-9.

28. Su S, Liu Q, Chen J, Chen F, He C, Huang D, et al. A positive feedback loop between mesenchymal-like cancer cells and macrophages is essential to breast cancer metastasis. Cancer Cell. 2014;25(5):605-20.

29. Brechbuhl HM, Finlay-Schultz J, Yamamoto TM, Gillen AE, Cittelly DM, Tan $A C$, et al. Fibroblast subtypes regulate responsiveness of luminal breast Cancer to estrogen. Clin Cancer Res. 2017;23(7):1710-21.
30. Fabris VT, Sahores A, Vanzulli SI, Colombo L, Molinolo AA, Lanari C, et al. Inoculated mammary carcinoma-associated fibroblasts: contribution to hormone independent tumor growth. BMC Cancer. 2010;10:293.

31. Giulianelli S, Cerliani JP, Lamb CA, Fabris VT, Bottino MC, Gorostiaga MA, et al. Carcinoma-associated fibroblasts activate progesterone receptors and induce hormone independent mammary tumor growth: a role for the FGF2/FGFR-2 axis. Int J Cancer. 2008;123(11):2518-31.

32. Turczyk L, Kitowska K, Mieszkowska M, Mieczkowski K, Czaplinska D, Piasecka $D$, et al. FGFR2-driven signaling counteracts tamoxifen effect on ERapositive breast Cancer cells. Neoplasia. 2017;19(10):791-804.

33. Pontiggia O, Sampayo R, Raffo D, Motter A, Xu R, Bissell MJ, et al. The tumor microenvironment modulates tamoxifen resistance in breast cancer: a role for soluble stromal factors and fibronectin through $\beta 1$ integrin. Breast Cancer Res Treat. 2012;133(2):459-71.

34. Yuan J, Liu M, Yang L, Tu G, Zhu Q, Chen M, et al. Acquisition of epithelialmesenchymal transition phenotype in the tamoxifen-resistant breast cancer cell: a new role for $\mathrm{G}$ protein-coupled estrogen receptor in mediating tamoxifen resistance through cancer-associated fibroblast-derived fibronectin and $\beta 1$-integrin signaling pathway in tumor cells. Breast Cancer Res. 2015;17:69.

35. Shekhar MP, Santner S, Carolin KA, Tait L. Direct involvement of breast tumor fibroblasts in the modulation of tamoxifen sensitivity. Am J Pathol. 2007;170(5):1546-60

36. Burke D, Wilkes D, Blundell TL, Malcolm S. Fibroblast growth factor receptors: lessons from the genes. Trends Biochem Sci. 1998;23(2):59-62.

37. Sleeman M, Fraser J, McDonald M, Yuan S, White D, Grandison P, et al. Identification of a new fibroblast growth factor receptor, FGFR5. Gene. 2001; 271(2):171-82

38. Babina IS, Turner NC. Advances and challenges in targeting FGFR signalling in cancer. Nat Rev Cancer. 2017;17(5):318-32.

39. Wesche J, Haglund K, Haugsten EM. Fibroblast growth factors and their receptors in cancer. Biochem J. 2011;437(2):199-213.

40. Brooks AN, Kilgour E, Smith PD. Molecular pathways: fibroblast growth factor signaling: a new therapeutic opportunity in cancer. Clin Cancer Res. 2012;18(7):1855-62.

41. Ornitz $\mathrm{DM}$, Itoh $\mathrm{N}$. The fibroblast growth factor signaling pathway. Wiley Interdiscip Rev Dev Biol. 2015;4(3):215-66.

42. Mailleux AA, Spencer-Dene B, Dillon C, Ndiaye D, Savona-Baron C, Itoh N, et al. Role of FGF10/FGFR2b signaling during mammary gland development in the mouse embryo. Development. 2002;129(1):53-60.

43. Parsa S, Ramasamy SK, De Langhe S, Gupte W, Haigh JJ, Medina D, et al. Terminal end bud maintenance in mammary gland is dependent upon FGFR2b signaling. Dev Biol. 2008;317(1):121-31.

44. Pond AC, Bin X, Batts T, Roarty K, Hilsenbeck S, Rosen JM. Fibroblast growth factor receptor signaling is essential for normal mammary gland development and stem cell function. Stem Cells. 2013;31(1):178-89.

45. Adnane J, Gaudray P, Dionne CA, Crumley G, Jaye M, Schlessinger J, et al. $B E K$ and FLG, two receptors to members of the FGF family, are amplified in subsets of human breast cancers. Oncogene. 1991;6(4):659-63.

46. Jaakkola S, Salmikangas P, Nylund S, Partanen J, Armstrong E, Pyrhönen S, et al. Amplification of fgfr4 gene in human breast and gynecological cancers. Int J Cancer. 1993;54(3):378-82.

47. Penault-Llorca F, Bertucci F, Adélaïde J, Parc P, Coulier F, Jacquemier J, et al. Expression of FGF and FGF receptor genes in human breast cancer. Int J Cancer. 1995;61(2):170-6.

48. Elbauomy Elsheikh S, Green AR, Lambros MB, Turner NC, Grainge MJ, Powe D, et al. FGFR1 amplification in breast carcinomas: a chromogenic in situ hybridisation analysis. Breast Cancer Res. 2007;9(2):R23.

49. Helsten T, Elkin S, Arthur E, Tomson BN, Carter J, Kurzrock R. The FGFR landscape in Cancer: analysis of 4,853 tumors by next-generation sequencing. Clin Cancer Res. 2016:22(1):259-67.

50. Bange J, Prechtl D, Cheburkin Y, Specht K, Harbeck N, Schmitt M, et al. Cancer progression and tumor cell motility are associated with the FGFR4 Arg (388) allele. Cancer Res. 2002;62(3):840-7.

51. Thussbas C, Nahrig J, Streit S, Bange J, Kriner M, Kates R, et al. FGFR4 Arg388 allele is associated with resistance to adjuvant therapy in primary breast cancer. J Clin Oncol. 2006:24(23):3747-55.

52. Fillmore CM, Gupta PB, Rudnick JA, Caballero S, Keller PJ, Lander ES, et al. Estrogen expands breast cancer stem-like cells through paracrine FGF/Tbx3 signaling. Proc Natl Acad Sci U S A. 2010;107(50):21737-42. 
53. Yu M, Bardia A, Aceto N, Bersani F, Madden MW, Donaldson MC, et al. Cancer therapy. Ex vivo culture of circulating breast tumor cells for individualized testing of drug susceptibility. Science. 2014;345(6193): 216-20.

54. Garcia-Murillas I, Schiavon G, Weigelt B, Ng C, Hrebien S, Cutts RJ, et al. Mutation tracking in circulating tumor DNA predicts relapse in early breast cancer. Sci Transl Med. 2015;7(302):302ra133.

55. Wang S, Ding Z. Fibroblast growth factor receptors in breast cancer. Tumour Biol. 2017:39(5):1010428317698370.

56. Cox DG, Curtit E, Romieu G, Fumoleau P, Rios M, Bonnefoi H, et al. GWAS in the SIGNAL/PHARE clinical cohort restricts the association between the FGFR2 locus and estrogen receptor status to HER2-negative breast cancer patients. Oncotarget. 2016;7(47):77358-64

57. Jia C, Cai Y, Ma Y, Fu D. Quantitative assessment of the effect of FGFR2 gene polymorphism on the risk of breast cancer. Breast Cancer Res Treat. 2010;124(2):521-8.

58. Zhou L, Yao F, Luan H, Wang Y, Dong X, Zhou W, et al. Three novel functional polymorphisms in the promoter of FGFR2 gene and breast cancer risk: a HuGE review and meta-analysis. Breast Cancer Res Treat. 2012; 136(3):885-97.

59. Cui F, Wu D, Wang W, He X, Wang M. Variants of FGFR2 and their associations with breast cancer risk: a HUGE systematic review and metaanalysis. Breast Cancer Res Treat. 2016;155(2):313-35.

60. Boyarskikh UA, Zarubina NA, Biltueva JA, Sinkina TV, Voronina EN, Lazarev $A F$, et al. Association of FGFR2 gene polymorphisms with the risk of breast cancer in population of West Siberia. Eur J Hum Genet. 2009;17:1688-91.

61. Marian C, Ochs-Balcom HM, Nie J, Kallakury BV, Ambrosone CB, Trevisan M, et al. FGFR2 intronic SNPs and breast cancer risk: associations with tumor characteristics and interactions with exogenous exposures and other known breast cancer risk factors. Int J Cancer. 2011;129(3):702-12.

62. Wang $H$, Yang $Z$, Zhang $H$. Assessing interactions between the associations of fibroblast growth factor receptor 2 common genetic variants and hormone receptor status with breast cancer risk. Breast Cancer Res Treat. 2013;137(2):511-22

63. Zhang Y, Lu H, Ji H, Lu L, Liu P, Hong R, et al. Association between rs11200014, rs2981579, and rs1219648 polymorphism and breast cancer susceptibility: a meta-analysis. Medicine (Baltimore). 2017;96(50):e9246.

64. Wu J, Wang Y, Liu J, Chen Q, Pang D, Jiang Y. Effects of FGFR1 gene polymorphisms on the risk of breast Cancer and FGFR1 protein expression. Cell Physiol Biochem. 2018;47(6):2569-78.

65. Ghoussaini M, French JD, Michailidou K, Nord S, Beesley J, Canisus S, et al. Evidence that the 5 p12 variant rs 10941679 confers susceptibility to estrogen-receptor-positive breast Cancer through FGF10 and MRPS30 regulation. Am J Hum Genet. 2016;99(4):903-11.

66. Tiong KH, Mah LY, Leong CO. Functional roles of fibroblast growth factor receptors (FGFRs) signaling in human cancers. Apoptosis. 2013;18(12):1447-68.

67. Garcia-Closas M, Hall P, Nevanlinna H, Pooley K, Morrison J, Richesson DA, et al. Heterogeneity of breast cancer associations with five susceptibility loci by clinical and pathological characteristics. PLoS Genet. 2008;4(4):e1000054.

68. Liang J, Chen P, Hu Z, Zhou X, Chen L, Li M, et al. Genetic variants in fibroblast growth factor receptor 2 (FGFR2) contribute to susceptibility of breast cancer in Chinese women. Carcinogenesis. 2008;29(12):2341-6.

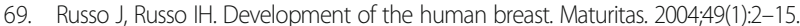

70. Brisken C, O'Malley B. Hormone action in the mammary gland. Cold Spring Harb Perspect Biol. 2010;2(12):a003178.

71. Owen Gl, Richer JK, Tung L, Takimoto G, Horwitz KB. Progesterone regulates transcription of the p21(WAF1) cyclin- dependent kinase inhibitor gene through Sp1 and CBP/p300. J Biol Chem. 1998;273(17):10696-701.

72. Stossi F, Madak-Erdogan Z, Katzenellenbogen BS. Estrogen receptor alpha represses transcription of early target genes via p300 and CtBP1. Mol Cell Biol. 2009;29(7):1749-59.

73. Mohammed H, Russell IA, Stark R, Rueda OM, Hickey TE, Tarulli GA, et al. Progesterone receptor modulates ERa action in breast cancer. Nature. 2015; 523(7560):313-7

74. Ballare C, Uhrig M, Bechtold T, Sancho E, Di Domenico M, Migliaccio A, et al. Two domains of the progesterone receptor interact with the estrogen receptor and are required for progesterone activation of the c-Src/Erk pathway in mammalian cells. Mol Cell Biol. 2003;23(6):1994-2008.

75. Denner LA, Weigel NL, Maxwell BL, Schrader WT, O'Malley BW. Regulation of progesterone receptor-mediated transcription by phosphorylation. Science. 1990;250(4988):1740-3.
76. Labriola L, Salatino M, Proietti CJ, Pecci A, Coso OA, Kornblihtt AR, et al Heregulin induces transcriptional activation of the progesterone receptor by a mechanism that requires functional ErbB-2 and mitogenactivated protein kinase activation in breast cancer cells. Mol Cell Biol. 2003;23(3):1095-111.

77. Cui X, Zhang P, Deng W, Oesterreich S, Lu Y, Mills GB, et al. Insulin-like growth factor-I inhibits progesterone receptor expression in breast cancer cells via the phosphatidylinositol 3-kinase/Akt/mammalian target of rapamycin pathway: progesterone receptor as a potential indicator of growth factor activity in breast cancer. Mol Endocrinol. 2003;17(4):575-88.

78. Daniel AR, Faivre EJ, Lange CA. Phosphorylation-dependent antagonism of sumoylation derepresses progesterone receptor action in breast cancer cells. Mol Endocrinol. 2007:21(12):2890-906.

79. Cerliani JP, Guillardoy T, Giulianelli S, Vaque JP, Gutkind JS, Vanzulli SI, et al. Interaction between FGFR-2, STAT5, and progesterone receptors in breast cancer. Cancer Res. 2011;71(10):3720-31.

80. Piasecka D, Kitowska K, Czaplinska D, Mieczkowski K, Mieszkowska M, Turczyk L, et al. Fibroblast growth factor signalling induces loss of progesterone receptor in breast cancer cells. Oncotarget. 2016;7(52):86011-25.

81. Ma ZQ, Santagati S, Patrone C, Pollio G, Vegeto E, Maggi A. Insulin-like growth factors activate estrogen receptor to control the growth and differentiation of the human neuroblastoma cell line SK-ER3. Mol Endocrinol. 1994;8(7):910-8.

82. Kato S, Endoh H, Masuhiro Y, Kitamoto T, Uchiyama S, Sasaki H, et al. Activation of the estrogen receptor through phosphorylation by mitogenactivated protein kinase. Science. 1995:270(5241):1491-4.

83. Ignar-Trowbridge DM, Nelson KG, Bidwell MC, Curtis SW, Washburn TF, McLachlan JA, et al. Coupling of dual signaling pathways: epidermal growth factor action involves the estrogen receptor. Proc Natl Acad Sci U S A. 1992; 89(10):4658-62.

84. Bunone G, Briand PA, Miksicek RJ, Picard D. Activation of the unliganded estrogen receptor by EGF involves the MAP kinase pathway and direct phosphorylation. EMBO J. 1996;15(9):2174-83.

85. Joel PB, Traish AM, Lannigan DA. Estradiol-induced phosphorylation of serine 118 in the estrogen receptor is independent of p42/p44 mitogenactivated protein kinase. J Biol Chem. 1998;273(21):13317-23.

86. Zhou W, Slingerland JM. Links between oestrogen receptor activation and proteolysis: relevance to hormone-regulated cancer therapy. Nat Rev Cancer. 2014;14(1):26-38.

87. Qiu M, Olsen A, Faivre E, Horwitz KB, Lange CA. Mitogen-activated protein kinase regulates nuclear association of human progesterone receptors. Mol Endocrinol. 2003;17(4):628-42.

88. Qiu M, Lange CA. MAP kinases couple multiple functions of human progesterone receptors: degradation, transcriptional synergy, and nuclear association. J Steroid Biochem Mol Biol. 2003:85(2-5):147-57.

89. Shen T, Horwitz KB, Lange CA. Transcriptional hyperactivity of human progesterone receptors is coupled to their ligand-dependent downregulation by mitogen-activated protein kinase-dependent phosphorylation of serine 294. Mol Cell Biol. 2001;21(18):6122-31.

90. Gee JM, Robertson JF, Gutteridge E, Ellis IO, Pinder SE, Rubini M, et al. Epidermal growth factor receptor/HER2/insulin-like growth factor receptor signalling and oestrogen receptor activity in clinical breast cancer. Endocr Relat Cancer. 2005:12(Suppl 1):S99-S111.

91. Knutson TP, Daniel AR, Fan D, Silverstein KA, Covington KR, Fuqua SA, et al. Phosphorylated and sumoylation-deficient progesterone receptors drive proliferative gene signatures during breast cancer progression. Breast Cancer Res. 2012;14(3):R95.

92. Abdel-Hafiz H, Dudevoir ML, Horwitz KB. Mechanisms underlying the control of progesterone receptor transcriptional activity by SUMOylation. J Biol Chem. 2009;284(14):9099-108.

93. Knutson TP, Truong TH, Ma S, Brady NJ, Sullivan ME, Raj G, et al. Posttranslationally modified progesterone receptors direct ligand-specific expression of breast cancer stem cell-associated gene programs. J Hematol Oncol. 2017;10(1):89.

94. McLeskey SW, Zhang L, El-Ashry D, Trock BJ, Lopez CA, Kharbanda S, et al. Tamoxifen-resistant fibroblast growth factor-transfected MCF-7 cells are cross-resistant in vivo to the antiestrogen $\mathrm{ICl} 182,780$ and two aromatase inhibitors. Clin Cancer Res. 1998;4(3):697-711.

95. Ruohola JK, Viitanen TP, Valve EM, Seppanen JA, Loponen NT, Keskitalo JJ, et al. Enhanced invasion and tumor growth of fibroblast growth factor $8 \mathrm{~b}-$ overexpressing MCF-7 human breast cancer cells. Cancer Res. 2001;61(10):4229-37. 
96. Turner N, Pearson A, Sharpe R, Lambros M, Geyer F, Lopez-Garcia MA, et al. FGFR1 amplification drives endocrine therapy resistance and is a therapeutic target in breast cancer. Cancer Res. 2010;70(5):2085-94.

97. Formisano L, Stauffer KM, Young CD, Bhola NE, Guerrero-Zotano AL, Jansen VM, et al. Association of FGFR1 with ERalpha maintains ligand-independent ER transcription and mediates resistance to estrogen deprivation in ER(+) breast Cancer. Clin Cancer Res. 2017;23(20):6138-50.

98. Itoh N, Ohta H. Fgf10: a paracrine-signaling molecule in development, disease, and regenerative medicine. Curr Mol Med. 2014;14(4):504-9.

99. Campbell TM, Castro MAA, de Santiago I, Fletcher MNC, Halim S, Prathalingam $\mathrm{R}$, et al. FGFR2 risk SNPs confer breast cancer risk by augmenting oestrogen responsiveness. Carcinogenesis. 2016;37(8):741-50.

100. Campbell TM, Castro MAA, de Oliveira KG, Ponder BAJ, Meyer KB. ERa binding by transcription factors NFIB and YBX1 enables FGFR2 signaling to modulate estrogen responsiveness in breast Cancer. Cancer Res. 2018;78(2):410-21.

101. Lanari C, Lamb CA, Fabris VT, Helguero LA, Soldati R, Bottino MC, et al. The MPA mouse breast cancer model: evidence for a role of progesterone receptors in breast cancer. Endocr Relat Cancer. 2009;16:333-50.

102. Santen RJ, Santner SJ, Pauley RJ, Tait L, Kaseta J, Demers LM, et al. Estrogen production via the aromatase enzyme in breast carcinoma: which cell type is responsible? J Steroid Biochem Mol Biol. 1997;61(3-6):267-71.

103. Busch S, Rydén L, Stål O, Jirström K, Landberg G. Low ERK phosphorylation in cancer-associated fibroblasts is associated with tamoxifen resistance in pre-menopausal breast cancer. PLoS One. 2012;7(9):e45669.

104. Kalluri R, Weinberg RA. The basics of epithelial-mesenchymal transition. J Clin Invest. 2009;119(6):1420-8.

105. Zhang L, Kharbanda S, Chen D, Bullocks J, Miller DL, Ding IY, et al. MCF-7 breast carcinoma cells overexpressing FGF-1 form vascularized, metastatic tumors in ovariectomized or tamoxifen-treated nude mice. Oncogene. 1997; 15(17):2093-108

106. McLeskey SW, Kurebayashi J, Honig SF, Zwiebel J, Lippman ME, Dickson RB, et al. Fibroblast growth factor 4 transfection of MCF-7 cells produces cell lines that are tumorigenic and metastatic in ovariectomized or tamoxifentreated athymic nude mice. Cancer Res. 1993;53(9):2168-77.

107. Giltnane JM, Hutchinson KE, Stricker TP, Formisano L, Young CD, Estrada MV et al. Genomic profiling of ER(+) breast cancers after short-term estrogen suppression reveals alterations associated with endocrine resistance. Sci Transl Med. 2017;9(402).

108. Tomlinson DC, Knowles MA, Speirs V. Mechanisms of FGFR3 actions in endocrine resistant breast cancer. Int J Cancer. 2012;130(12):2857-66.

109. Meijer D, Sieuwerts AM, Look MP, van Agthoven T, Foekens JA, Dorssers LC. Fibroblast growth factor receptor 4 predicts failure on tamoxifen therapy in patients with recurrent breast cancer. Endocr Relat Cancer. 2008;15(1):101-11.

110. Brady N, Chuntova P, Bade LK, Schwertfeger KL. The FGF/FGFR axis as a therapeutic target in breast cancer. Expert Rev Endocrinol Metab. 2013;8(4): 391-402.

111. Katoh M. Fibroblast growth factor receptors as treatment targets in clinical oncology. Nat Rev Clin Oncol. 2019;16(2):105-22.

112. André F, Bachelot T, Campone M, Dalenc F, Perez-Garcia JM, Hurvitz SA, et al. Targeting FGFR with dovitinib (TKI258): preclinical and clinical data in breast cancer. Clin Cancer Res. 2013;19(13):3693-702.

113. Musolino A, Campone M, Neven P, Denduluri N, Barrios CH, Cortes J, et al. Phase $\mathrm{II}$, randomized, placebo-controlled study of dovitinib in combination with fulvestrant in postmenopausal patients with HR(+), HER2(-) breast cancer that had progressed during or after prior endocrine therapy. Breast Cancer Res. 2017;19(1):18.

114. Cheng FT, Ou-Yang F, Lapke N, Tung KC, Chen YK, Chou YY, et al. Pazopanib sensitivity in a patient with breast Cancer and FGFR1 amplification. J Natl Compr Cancer Netw. 2017;15(12):1456-9.

115. Matsui J, Funahashi Y, Uenaka T, Watanabe T, Tsuruoka A, Asada M. Multikinase inhibitor E7080 suppresses lymph node and lung metastases of human mammary breast tumor MDA-MB-231 via inhibition of vascular endothelial growth factor-receptor (VEGF-R) 2 and VEGF-R3 kinase. Clin Cancer Res. 2008;14(17):5459-65.

116. Chae YK, Ranganath K, Hammerman PS, Vaklavas C, Mohindra N, Kalyan A, et al. Inhibition of the fibroblast growth factor receptor (FGFR) pathway: the current landscape and barriers to clinical application. Oncotarget. 2017;8: 16052-74.

117. Smyth EC, Turner NC, Pearson A, Peckitt C, Chau I, Watkins DJ, et al. Phase II study of AZD4547 in FGFR amplified tumours: gastroesophageal cancer (GC) cohort pharmacodynamic and biomarker results. J Clin Oncol. 2016;34(4) (Suppl):154.

118. Nogova L, Sequist LV, Perez Garcia JM, Andre F, Delord JP, Hidalgo M, et al. Evaluation of BGJ398, a fibroblast growth factor receptor 1-3 kinase inhibitor, in patients with advanced solid tumors harboring genetic alterations in fibroblast growth factor receptors: results of a global phase I, dose-escalation and dose-expansion study. J Clin Oncol. 2017;35(2):157-65.

119. Harding TC, Long L, Palencia S, Zhang H, Sadra A, Hestir K, et al. Blockade of nonhormonal fibroblast growth factors by FP-1039 inhibits growth of multiple types of cancer. Sci Transl Med. 2013;5(178):178ra39.

120. Donnell O, Goldman PJW, Gordon MS, Shih K, Choi YJ, Lu D, et al. A phase I dose-escalation study of MFGR1877S, a human monoclonal anti-fibroblast growth factor receptor 3 (FGFR3) antibody, in patients (pts) with advanced solid tumors. Eur J Cancer. 2012;48:191-2.

121. Gemo AT, Deshpande AM, Palencia S, Bellovin DI, Brennan TJ, Patil NS, et al. FPA144: a therapeutic antibody for treating patients with gastric cancers bearing FGFR2 gene amplification. Cancer Res. 2014;74(Suppl:5446).

\section{Ready to submit your research? Choose BMC and benefit from:}

- fast, convenient online submission

- thorough peer review by experienced researchers in your field

- rapid publication on acceptance

- support for research data, including large and complex data types

- gold Open Access which fosters wider collaboration and increased citations

- maximum visibility for your research: over $100 \mathrm{M}$ website views per year

At $\mathrm{BMC}$, research is always in progress.

Learn more biomedcentral.com/submissions 